\title{
Pulmonary hypertension due to micro-thromboembolism from splenic and portal veins after portacaval anastomosis
}

\author{
M. Sallam and W. C. Watson \\ From the Gastro-intestinal Unit, Glasgow Royal Infirmary
}

This is a presentation of a unique case of pulmonary hypertension in a 15-year-old girl, due to micro-thromboembolism from splenoportal veins through a portacaval anastomosis.

Micro-thromboembolism producing insidious pulmonary hypertension is an important category of pulmonary embolism. It contrasts with other types which, because the emboli are larger, present with acute or subacute symptoms. Wood (1956) in his description of subacute thromboembolic pulmonary hypertension stressed the importance of early diagnosis and treatment with anticoagulants. Goodwin (1960) pointed out that thromboembolism was probably the commonest cause of obliterative pulmonary vascular disease. $\mathrm{He}$ and his colleagues (Goodwin, Harrison, and Wilcken, 1963) classified patients with chronic pulmonary hypertension of embolic origin into two groups, those with large emboli affecting main or segmental arteries, and others with small emboli affecting microscopic vessels leading to progressive obliterative thromboembolic pulmonary hypertension. A more recent review (Fleischner, 1967) separates the groups on a clinical as well as radiological basis into those with an acute onset of chest pain, dyspnoea, and haemoptysis, and those with insidious onset of pulmonary hypertension who are likely to be diagnosed as idiopathic pulmonary hypertension.

The source of pulmonary embolism is thrombus which is usually in the leg veins, occasionally in the pelvic veins, and rarely in the heart in conditions such as endocardial fibrosis or fibroelastosis, or after myocardial infarction affecting the right ventricle. Bedrest, immobilization, trauma to legs or pelvis, malignancy, oral contraceptives, heart failure, and atrial fibrillation are important factors. The incidence is increased after the age of 20 .
It is doubtful if age by itself is an important factor in the aetiology of thromboembolism (Marshall, 1965), though the incidence is particularly low in children.

\section{Case report}

This is an account of a unique case of pulmonary hypertension secondary to thromboembolism arising in the portal and splenic veins of a 15year-old girl, after a portacaval anastomosis.

The patient presented at the age of 5 with haematemesis and melaena from oesophageal varices. She had hepatosplenomegaly, and the diagnosis of Banti's syndrome was made. She continued to have episodes of acute alimentary bleeding, until at the age of 9 a portacaval anastomosis was performed. At operation the distal half of the portal vein was seen to be varicosed and thrombosed, and there was some loose clot. Liver biopsy showed no evidence of cirrhosis. After operation there was considerable involution of the spleen and the oesophageal varices disappeared. There was no further trouble with anaemia or bleeding and her growth and development proceeded normally.

At a review medical examination at the age of I5 there were clinical signs of pulmonary hypertension and on direct questioning the patient admitted to increasing breathlessness over a period of ro months, without cough, chest pain, or haemoptysis. A provisional diagnosis of thromboembolic pulmonary hypertension was made and she was admitted for investigation.

She was a well-developed girl without cyanosis, pallor, or finger clubbing. The external jugular vein filled to $5 \mathrm{~cm}$. above the angle of the sternum, and there was a prominent $A$ wave. The pulse was regular at $68 / \mathrm{min}$. BP $130 / 70$ $\mathrm{mm}$. Hg. The apex beat was in the 5 th interspace in the midclavicular line. There was a right ventricular heave over the left parasternal area. The 
first sound at the pulmonary area was normal and followed by an ejection systolic murmur grade $3 / 6$. The second sound at the same area was narrowly split, with accentuation of the pulmonary component.

The liver and spleen were enlarged $3-4 \mathrm{~cm}$. and were firm. The chest and central nervous system were normal.

Investigations $\mathrm{Hb} \quad 13.8 \quad \mathrm{~g} . / 100 \mathrm{ml}$; WBC $3800 / \mathrm{cu}$. mm.; platelets $108,000 / \mathrm{cu}$. mm.; reticulocytes $5 \%$; urea, electrolytes, standard liver function tests, and coagulation screen were all normal. Electrocardiogram showed moderate right ventricular hypertrophy. Chest $x$-ray showed slight enlargement of the heart, with prominence of the pulmonary artery. Pulmonary function tests were normal and, in particular, there was no evidence of alveo-capillary block.

Cardiac catheterization and pulmonary angiography were performed (Fig. I). The pulmonary artery pressure was $70 / 32 \mathrm{~mm}$. $\mathrm{Hg}$, with a mean pressure of $48 \mathrm{~mm}$. $\mathrm{Hg}$. There was no obliteration of major arteries or evidence of a left-sided heart lesion or left-to-right shunt.

A splenic venogram (Fig. 2) showed the portocaval anastomosis to be widely patent. Pressure in the splenic and portal veins was normal, but a movable small mass, probably thrombus, was observed in the veins at the hilum of the spleen.

\section{Discussion}

This case represents the very rare situation in which embolus arising in the portal vein could reach the lung through a portacaval anastomosis and lead to obstructive pulmonary hypertension. The diagnosis was made initially on the positive factors compatible with it and corroborated by exclusion of other causes of pulmonary hypertension. Post-capillary (passive) hypertension secondary to left-sided heart lesions such as mitral stenosis, mitral incompetence, and atrial myxoma as well as stenosis of the pulmonary veins was excluded by angiography. The so-called high output states of anaemia and thyrotoxicosis were also absent, and even liver cirrhosis was excluded by liver biopsy. No parenchymal lung disease could be shown clinically, radiologically, or by pulmonary function tests. There were no features to suggest those rare types of pulmonary vascular disease found in association with systemic lupus erythematosus or periarteritis nodosa. We are aware that cardiac output is increased after portacaval anastomosis in human patients (Even et al., 1965) as well as after experimental surgery in dogs (Johnson and Lambert, 1967). This increase by itself is probably insufficient to cause a moderately severe pulmonary hypertension. The diagnosis of primary or idiopathicpulmonary

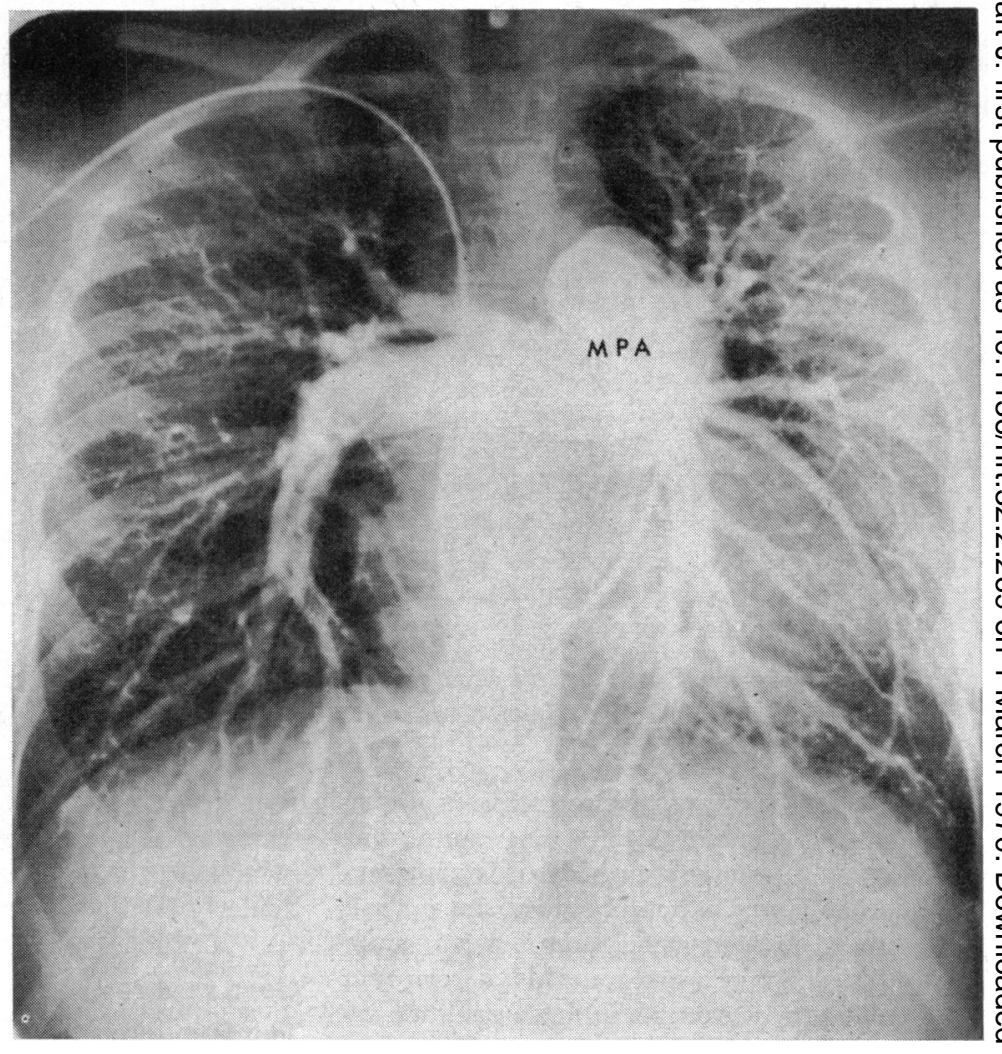

FIG. I Pulmonary angiogram showing enlarged main pulmonary artery (MPA). There is some diminution in the extension of the small vessels, particularly at both lower zones.

hypertension is made with diminishing frequency as other causes of pulmonary hypertension are discovered (Friedberg, 1966).

In the patient reported here the site of origin of the pulmonary emboli was accepted as being in the portal and splenic veins and their tributaries. Evidence supporting this conclusion was the operation report of portal vein pathology manifest as tortuosity and thrombosis, plus the recent venogram showing thrombus at the hilum of the spleen.

Treatment and prognosis Anticoagulants are indicated as early as possible in patients with recurrent and subacute thromboembolism, and before obliterative lesions in the pulmonary vasculature are well established. Wood (1956) regarded anticoagulant therapy as worth while, especially if given early in the course of the disease. Thompson and Hamilton (1962) and Goodwin et al. (1963) were, on the other hand, less enthusi- 
astic about anticoagulant therapy, since in their experience progressive obliterative vascular disease eventually led to right heart failure in both treated and untreated patients, though there was a slightly better prognosis in the treated cases.

It seems a reasonable premise nevertheless that better results should be expected if anticoagulants are given before well-established obliterative pulmonary hypertension has occurred.

Splenectomy was not advised in this patient at the present time for two reasons. First, the mild degree of hypersplenism present was not sufficient to warrant splenectomy, with its possible sequel of an increase in platelet count. Secondly the splenic vein was probably not the only source of embolism; the portal vein and its tributaries were also likely contributors.

One aspect of this case is puzzling and that is why in the course of six years' exposure to the risk of pulmonary embolism this patient never had at least one embolism large enough to cause overt symptoms. The possible answer may be that chronic thrombocytopenia has meant that whatever thrombus was produced in the splenoportal veins was of poor quality and readily broken into microemboli.

We are indebted to Dr. Moran of the Cardiorespiratory Unit and Drs. Moule and McKellar of the Department of Radiology for the special investigations.

\section{References}

Even, P., Nicollo, F., Benhamou, J. P., and Fauvert, R. (1965). Le débit cardiaque au cours des maladies du foie. Effets de l'anastomose porto-cave et des diurétiques. Revue Franfaise d'Études Cliniques et Biologiques, ro, 799.

Fleischner, F. G. (1967). Recurrent pulmonary embolism and cor pulmonale. New England fournal of Medicine, 276, 1213.

Friedberg, C. K. (1966). Diseases of the Heart, 3 rd ed. Saunders, Philadelphia.

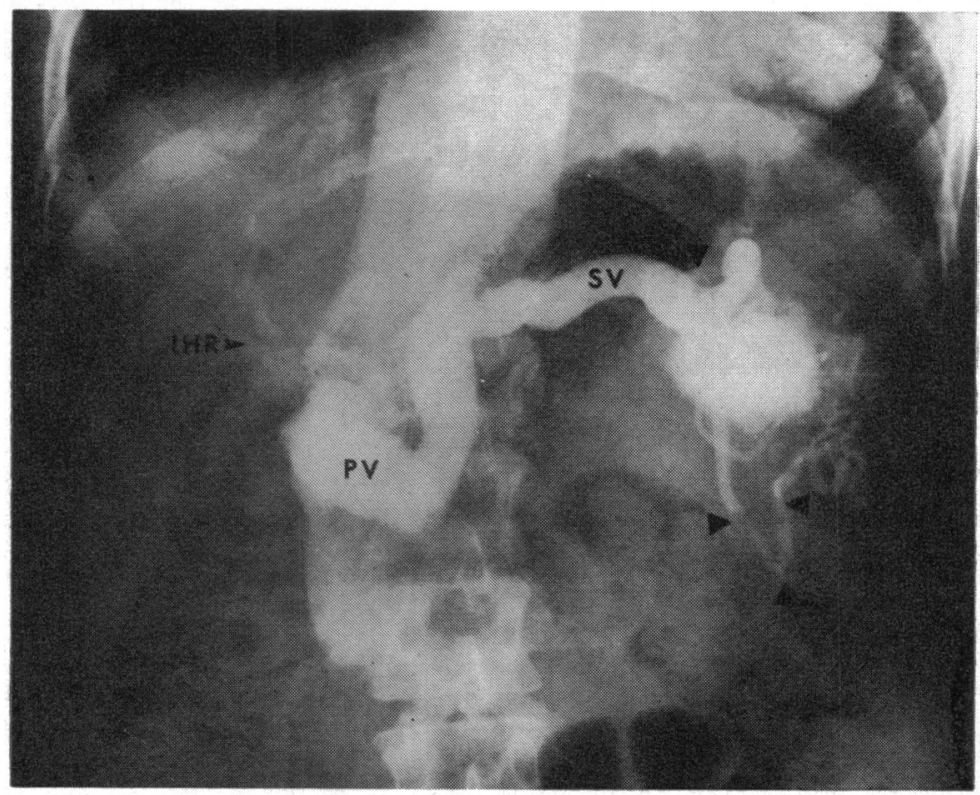

FIG. 2 Splenogram showing a filling defect in the splenic vein close to the hilum suggesting a thrombus (upper arrow). The lower arrows show further filling defects in a vein at the lower pole of the spleen. These are probably also thrombi. SV, splenic vein; $P V$, portal vein; IHR, varicose intrahepatic portal radicles.

Goodwin, J. F. (1960). The nature of pulmonary hypertension. In Clinical Disorders of the Pulmonary Circulation, p. 57. Ed. by R. Daley, J. F. Goodwin, and R. E. Steiner. Churchill, London.

, Harrison, C. V., and Wilcken, D. E. L. (1963) Obliterative pulmonary hypertension and thromboembolism. British Medical fournal, r, 701 and 777.

Johnson, G., and Lambert, J. (1967). Cardiac output after portacaval shunt. Annals of Surgery, 166, 207.

Marshall, R. (1965). Pulmonary Embolism-Mechanism and Management. Thomas, Springfield, Illinois.

Thompson, E. N., and Hamilton, M. (1962). Pulmonary-embolic disease. Lancet, 1, 1369.

Wood, P. (1956). Diseases of the Heart and Circulation, and ed. Eyre and Spottiswoode, London. 